\title{
Levantamento sazonal de nematódeos gastrointestinais em um rebanho ovino leiteiro
}

\author{
Seasonal survey of gastrointestinal nematodes in a milk sheep flock \\ Estudio estacional de nematodos gastrointestinales em um rebaño de oveja de leche
}

Recebido: 25/02/2021 | Revisado: 07/03/2021 | Aceito: 11/03/2021 | Publicado: 18/03/2021

\author{
Thaís Moreira Osório \\ ORCID: https://orcid.org/0000-0003-3172-2412 \\ Universidade Federal do Pampa, Brasil \\ E-mail: thais-osorio@uergs.edu.br \\ Leonardo de Melo Menezes \\ ORCID: https://orcid.org/0000-0001-8536-0803 \\ Universidade Estadual do Rio Grande do Sul, Brasil \\ E-mail: leonardo-menezes@uergs.edu.br \\ Karoline Barcellos da Rosa \\ ORCID: https://orcid.org/0000-0002-4890-4696 \\ Universidade Estadual do Rio Grande do Sul, Brasil \\ E-mail: karoline-rosa@uergs.edu.br \\ Rodrigo Flores Escobar \\ ORCID: https://orcid.org/0000-0003-1548-512X \\ Universidade Estadual do Rio Grande do Sul, Brasil. \\ E-mail: rodrigo-escobar@uergs.edu.br \\ Rivas Matheus Lencina dos Santos \\ ORCID: https://orcid.org/0000-0001-9323-9149 \\ Universidade Estadual do Rio Grande do Sul, Brasil \\ E-mail: rivas-santos@uergs.edu.br \\ Gianny de Mello Maydana \\ ORCID: https://orcid.org/0000-0001-6237-3695 \\ Universidade Estadual do Rio Grande do Sul, Brasil \\ E-mail: gianny-maydana@uergs.edu.br \\ Velci Queiroz de Souza \\ ORCID: https://orcid.org/0000-0002-6890-6015 \\ Universidade Federal do Pampa, Brasil \\ E-mail: velcisouza@unipampa.edu.br
}

\section{Resumo}

Foi estudada a epidemiologia dos nematódeos gastrintestinais em 120 ovinos pertencentes às raças Lacaune, Crioulas e mestiças destas duas, mantidas em regime semi-intensivo de pastoreio em uma propriedade particular, no município de Santana do Livramento, Fronteira Oeste do Rio Grande do Sul. O clima predominante da região é o Cfa e o experimento foi conduzido por 12 meses, englobando as quatro estações climáticas do ano. Os animais eram portadores de infecção natural por diferentes espécies de helmintos e o trabalho baseou-se na contagem de ovos por grama de fezes de 30 animais, escolhidos aleatoriamente, e destes, realizou-se um pool de fezes das 10 amostras mais infectadas para realização da coprocultura, com o intuito de indicar os gêneros mais prevalentes nestas amostras. Os dados foram analisados utilizando o teste de Análise de Variância e as médias comparadas por meio do Teste de Scott-Knott, a um nível de significância de 5\%. Os gêneros de nematódeos mais prevalentes foram Haemonchus sp. e Trichostrongylus sp. Os dados de contagem de ovos por grama de fezes (OPG) indicaram que o ápice de produção de ovos foi na primavera. Além disso, observou-se a tendência de resistência desses helmintos encontrados aos fármacos utilizados para controle nesta propriedade. Conclui-se que há predominância de alguns gêneros de nematódeos no rebanho avaliado, e que existe diferença na distribuição epidemiológica em função da estação do ano.

Palavras-chave: Endoparasitoses; Epidemiologia; Estações do ano; Haemonchus sp.; Helmintos; Trichostrongylus sp.

\begin{abstract}
The epidemiology of gastrointestinal nematodes was studied in 120 breeds sheep of races Lacaune, Creole and their crosses in a semi-intensive grazing regime in private property, in Santana do Livramento, West Frontier of Rio Grande so Sul. The climate in the region is Cfa and the experimente was conducted for twelve months, crossing for four seasons of the year. The animals had natural infection by distincts species of helminths and the work was based on the egg count per gramo f feces (OPG) of 30 animals, on average, and these, made up a pool of faces of the tem most infected for coproculture, in order to indicate the most prevalent generas in these samples. The data were analyzed using the Analysis of Variance test and the means were compared using the Skott-Knott test, a significance level of 5\%. The most prvalent genera was Haemonchus sp. OPG data indicates that the peak of eggs production was
\end{abstract}


in the spring. In addition, there was a tendency for parasites to resist anthelmintics in this property. In conclusion, there are more prevalente nematódeos generas in sheep farming avaible and was observed difference in the epidemiological distribution according to the season.

Keywords: Endoparasitosis; Epidemiology; Haemonchus sp.; Helminths; Seasonal variation; Trichostrongylus sp.

\section{Resumen}

Se estudió la epidemiologia de los nematodos gastrointestinales en 120 ovejas pertenecientes a las razas lacaune, criolla y mestiza de estas dos mantenidas en régimen de pastoreo semi-intensivo en uma propiedad privada, en el ciudad de Santana do Livramento, Frontera Oeste de Rio Grande do Sul. El clima predominante de la región es Cfa y el experimento se desarrolló durante 12 meses, corriendo las cuatro estaciones climáticas del año. Los animales tenían infección natural por diferentes espécies de helmintos y el trabajo de basó en el conteo de huevos por gramo de heces de 30 animales, elegidos al azar, y de estos se realizo um pool de heces de las 10 muestras más infectadas para coprocultiva, con el fin de indicar los géneros más prevalentes em estas muestras. Los datos se analizaron mediante la prueba de Análisis de varianza unidirecional y las medias se compararon mediante la prueba de Skott-Knott, a um nível de significância del 5\%. Los géneros de nematodos más prevalentes fueron Haemonchus sp. y Trichostrongylus sp. Los datos del recuento de huevos por gramo de heces (OPG) indicaron que el pico de producción de huevos fue en la primavera. Además, se observo la tendencia de resistencia de estos helmintos a las drogas utilizadas para el control de esta propiedad. Se concluye que hay predominio de algunos géneros de nematodos en el rebaño evaluado, y que existe diferencia en la distribución epidemiológica según la época.

Palabras clave: Endoparasitosis; Estaciones del año; Epidemiología; Haemonchus sp.; Helmintos; Trichostrongylus sp.

\section{Introdução}

A ovinocultura é uma atividade importante para a para produção de carne, leite e peles, incrementando à economia do Estado do Rio Grande do Sul (Vieira, 2008). No Brasil, de acordo com dados do Instituto Brasileiro de Geografia e EstatísticaIBGE (2018), o efetivo total do rebanho ovino é de aproximadamente 18 milhões cabeças.

Os ovinos são susceptíveis à ação de nematódeos gastrintestinais, sendo as infecções causadas por estes o principal fator limitante desse setor pecuário, principalmente em regiões tropicais (Aquino et al., 2016, Leme et al., 2020, Osório et al., 2020). Isto porque são responsáveis por promover desnutrição, avitaminose, distúrbios gastrointestinais, estados convulsivos, comprometimento do desenvolvimento animal e anemia (Souza, 2013).

São importantes endoparasitas os seguintes gêneros da família Trichostrongilidae: Haemonchus contortus (abomaso), Trichostrongylus colubriformis (intestino delgado), Ostertagia circumcincta (abomaso) e Cooperia spp. (intestino delgado); da família Ancylostomatidae: Bunostomum spp. (intestino delgado); e da família Cyatostomidae destaca-se: Oesophagostomum spp. (intestino grosso) (Amarante et al., 2009, Silva et al., 2017, Roberto et al., 2018).

Na região sul do Brasil, os parasitas prevalentes na população de ovinos são da espécie Haemonchus contortus, que, em alguns casos, pode representar 99,99\% do parasitismo, seguido de Trichostrongylus colubriformis, Ostertagia ostertagi, Cooperia curticei, Nematodirus spatigher, Oesophagostomum venulosum e Trichuris ovis (Ramos et al., 2004, Menezes et al, 2011, Domingues et al., 2013).

O aparecimento destes endoparasitas ocorre, geralmente, devido ao manejo inadequado do rebanho, más condições sanitárias e uso indevido dos medicamentos existentes (Costa et al., 2017; Mottin et al., 2019). O controle mais comumente utilizado para atenuar a parasitose gastrointestinal nos rebanhos é a dose via oral de anti-helmínticos (Lacerda et al., 2010). No entanto, atualmente, há um agravante às parasitoses em ovinos, a resistência anti-helmíntica (Osório et al., 2020). Esta é agravada, em muitos casos, pela prática de vermifugação realizada sem critérios técnicos pelos criadores, principalmente em relação às dosagens e a frequência de uso (Cardoso et al., 2015). Consequentemente, o uso indiscriminado dos vermífugos acelera o aparecimento da resistência, aumenta os custos do produtor e ainda aumenta o volume de resíduos químicos nos produtos de origem animal tornando-se um risco à saúde pública e ao ecossistema (Tariq et al., 2008). Portanto, o uso indiscriminado de anti-helmínticos contribui para o surgimento de endopasitas resistentes (Salgado \& Santos, 2016). 
A fim de garantir melhor resultado ao tratamento utilizado, torna-se necessário avaliar previamente o anti-helmíntico por meio de testes de eficácia e, assim, instituir um controle eficaz (Cezar et al., 2010, Nova et al., 2014). Para Kate (1965), a qual já preconizava a importância de se conhecer o potencial biótico das helmintoses de ruminantes, quando estudadas e bem conhecidas em determinada região e período, torna-se viável estabelecer o seu modelo estacional, visando as dosificações estratégicas e práticas de controle. Por isso, o objetivo deste trabalho foi realizar um levantamento epidemiológico das helmintoses gastrintestinais e verificar a prevalência dos endoparasitas em diferentes sazonalidades, bem como a sensibilidade destes parasitas aos fármacos comercialmente disponíveis atualmente, afim de caracterizar esta problemática estabelecida na criação de ovinos.

\section{Metodologia}

Esta pesquisa tem perfil laboratorial, com material coletado em campo, sendo quali-quantitativa, pois além da mensuração numérica de dados, objetiva também a caracterização da problemática da resistência aos vermífugos disponíveis para enfrentamento às endoparasitoses na ovinocultura. Para fins metodológicos, este estudo baseou-se em Pereira et al. (2018).

\subsection{Caracterização da área de estudo}

Santana do Livramento é um município do estado do Rio Grande do Sul, no Brasil. Localiza-se a uma latitude

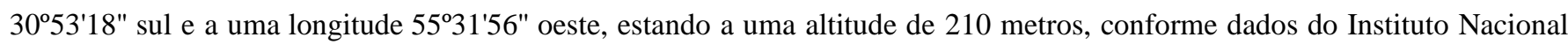
de Meteorologia. A temperatura média anual do município situa-se em torno de $18^{\circ} \mathrm{C}$ (INMET, In: Burial et al., 2019) e a precipitação pluviométrica média anual é de aproximadamente 1400 mm (Mena, 2017). Faz parte da região da Fronteira Oeste, destacando-se na pecuária, apresentando 344.785 ovinos, conforme IBGE (2017). O clima da região é subtropical úmido (Cfa), conforme classificação climática de Koeppen (Moreno, 1961).

\subsection{Propriedade rural e animais estudados}

A propriedade situa-se na região do munícipio denominada Cerro da Vigia, tendo como atividades a vitivinicultura, olericultura e criação de ovelhas leiteiras e de corte. O rebanho de ovinos leiteiros consiste em 120 animais, pertencentes às raças Lacaune, Crioulas e suas cruzas, mantidas em regime semi-intensivo de criação. As visitas à propriedade ocorreram entre os meses de abril de 2019 e março de 2020. Os animais foram manejados sob pastagens nativas e cultivadas durante o período diurno, sendo recolhidos à noite em um galpão, onde recebiam alimentação à base de feno de alfafa, silagem de milho e sal mineral.

\subsection{Coleta e processamento das amostras}

As amostras de fezes foram coletadas em uma amostragem de aproximadamente 30 ovinos por levantamento, individualmente, escolhidos aleatoriamente, por meio do sorteio dos brincos afixados nos animais. As mesmas foram realizadas sempre pelo período matutino em duas oportunidades, a cada estação do ano (aproximadamente 45 dias), sendo a primeira no começo e a segunda no meio da estação.

O material fecal era coletado diretamente da ampola retal dos animais, utilizando-se sacos plásticos descartáveis, devidamente identificados e adequadamente transportados até o Laboratório de Microbiologia e Microscopia da Universidade Estadual do Rio Grande do Sul (UERGS). Somente após a coleta os mesmos eram dosificados. A via de aplicação bem como as dosagens dos anti-helmínticos seguiu a recomendação indicada pelo fabricante. O dia da primeira coleta e aplicação dos anti-helmínticos foi considerado o dia zero (D0) e o retorno, após sete dias foi considerado o dia sete (D7), no qual realizou-se 
nova coleta dos mesmos animais, para aferição da eficácia do fármaco utilizado. A contagem de ovos por grama de fezes (OPG) foi realizada por meio da técnica de Gordon e Whitlock (1939), adaptada por Ueno e Gonçalves (1998). Já para a coprocultura, a técnica utilizada foi a adaptada de Roberts e O'Sullivan (1950), na qual era realizado um pool das fezes mais infectadas de cerca de 10 destes 30 animais, cujas fezes estavam mais infectadas no teste de OPG. A coprocultura permite identificar qualitativamente os parasitas presentes no rebanho (Ueno, 1998), a partir da identificação das larvas infectantes de terceiro estádio (L3), de acordo com as características morfológicas descritas por Keith (1953). Os animais foram submetidos à nova coleta de fezes sete dias após a dosificação com o anti-helmíntico, para estimar a eficácia dos fármacos aplicados.

\subsection{Análise dos resultados}

Os resultados foram analisados conforme o teste de Redução da Contagem de Ovos nas Fezes (RCOF), através da Equação:

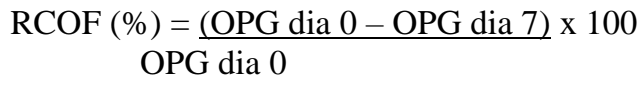

Em que o OPG médio pré-tratamento foi o obtido no D0 (dia zero), antes da aplicação do vermífugo e o OPG médio pós-tratamento, o resultante das amostragens realizadas no D7 (sete dias após aplicação do fármaco). Como indicativo de eficiência, foi considerado o que preconiza o teste de eficácia dos fármacos, verificado pelo teste de redução do número de ovos por grama de fezes (Edwards et al., 1986), calculada pela fórmula: \% eficácia = 1 - [(OPG médio pós-tratamento/OPG médio pré - tratamento)] x 100, indicando eficácia com índice maior ou igual a 95\% (Coles, 1992),

\subsection{Informações climáticas}

Os dados referentes às temperaturas médias e índices pluviométricos da região da Propriedade foram fornecidos pelo Instituto Nacional de Meteorologia (INMET), referentes a todos os meses em que ocorreram as análises e estes foram tabulados sempre a partir de 45 dias de antecedência até a data de cada um dos levantamentos realizados para o OPG no dia zero (D0), com o intuito de correlacionar os fatores ambientais e a possível influência destes para com os resultados parasitológicos.

\subsection{Análises Estatísticas}

Utilizou-se o delineamento inteiramente casualizado. Os dados foram analisados estatisticamente pelos procedimentos da análise de variância e o agrupamento de médias comparados pelo teste de Skott-Knott a 5\% de significância (P>0,05), utilizando o Genes (aplicativo computacional em estatística aplicada à genética).

\section{Resultados e Discussão}

Com os dados referentes às coletas de amostras de fezes dos ovinos, obtivemos uma média da infecção inicial no rebanho estudado a cada amostragem (OPG médio D0) e buscou-se correlacionar às condições meteorológicos na localidade, como mostra a Tabela 1. 
Tabela 1. Dados meteorológicos em cada levantamento amostral.

\begin{tabular}{lccccc}
\hline $\begin{array}{l}\text { Estações } \\
\text { do Ano }\end{array}$ & $\begin{array}{c}\text { OPGmédio } \\
\text { Do }\end{array}$ & $\begin{array}{c}\text { Índice pluviométrico } \\
(\mathbf{m m})\end{array}$ & $\begin{array}{c}\text { Temperatura } \\
\text { média }^{\circ} \boldsymbol{C}\end{array}$ & $\begin{array}{c}\text { Temperatura } \\
\text { mínima }^{\circ} \boldsymbol{C}\end{array}$ & $\begin{array}{c}\text { Temperatura } \\
\text { máxima }^{\circ} \boldsymbol{C}\end{array}$ \\
\hline Outono & 1100 & 81 & 20.37 & 14.79 & 27.19 \\
\hline Inverno & 2900 & 250 & 17.23 & 12.8 & 22.61 \\
\hline Primavera & 1800 & 90 & 15.56 & 11.5 & 20.83 \\
\hline & 1500 & 232 & 11.81 & 7.02 & 17.77 \\
\hline Verão & 15500 & 114 & 13.43 & 7.34 & 20.3 \\
\hline & 8500 & 184 & 18.19 & 12.95 & 24.23 \\
\hline
\end{tabular}

Fonte: Autores.

De acordo com os dados acima, verificou-se a flutuação dos dados da infecção parasitária, conforme alteração nas condições ambientais. Destacando-se, o aumento significativo do OPG médio inicial nas estações primavera e verão, na propriedade do estudo. Com isso, buscou-se, por meio das Figuras 1 e 2, observar a existência de correlação entre esses dados da infecção inicial (OPG médio no D0) e os fatores ambientais, índice pluviométrico e temperaturas.

Figura 1. Relação entre OPG (eixo X) e pluviosidade em mm (eixo Y) em ovinos leiteiros - entre abril de 2019 e março de 2020 .

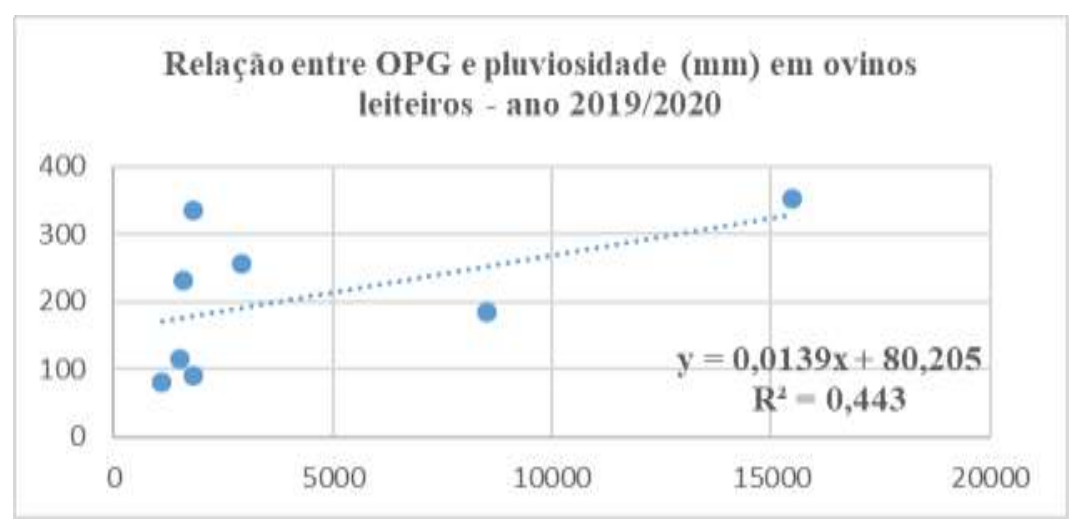

Fonte: Autores.

De acordo com à análise de regressão, houve correlação de 0,443 entre o OPG inicial médio e o índice pluviométrico médio para os 45 dias que antecederam a coleta, na localidade. O que demonstra correlação entre a pluviosidade e os maiores índices de infecção parasitária, algo que ficou mais evidente na primavera, período que os índices pluviométricos foram mais elevados, conforme demonstrado na Figura 1. Isso vem de encontro com o relato feito por Braga (1986) e Heck (2005), que afirmaram que a contaminação dos animais com formas parasitárias infectantes apresenta variação decorrente do microclima, sendo o índice pluviométrico, o principal fator responsável pelo aumento dessas contaminações.

A fim de também verificar a correlação da infecção inicial nas amostragens com às temperaturas, abaixo a Figura 2 permite a visualização dos resultados quanto à esta análise. 
Figura 2. Relação entre OPG (eixo X) e temperatura (eixo Y) em ovinos leiteiros - entre abril de 2019 e março de 2020.

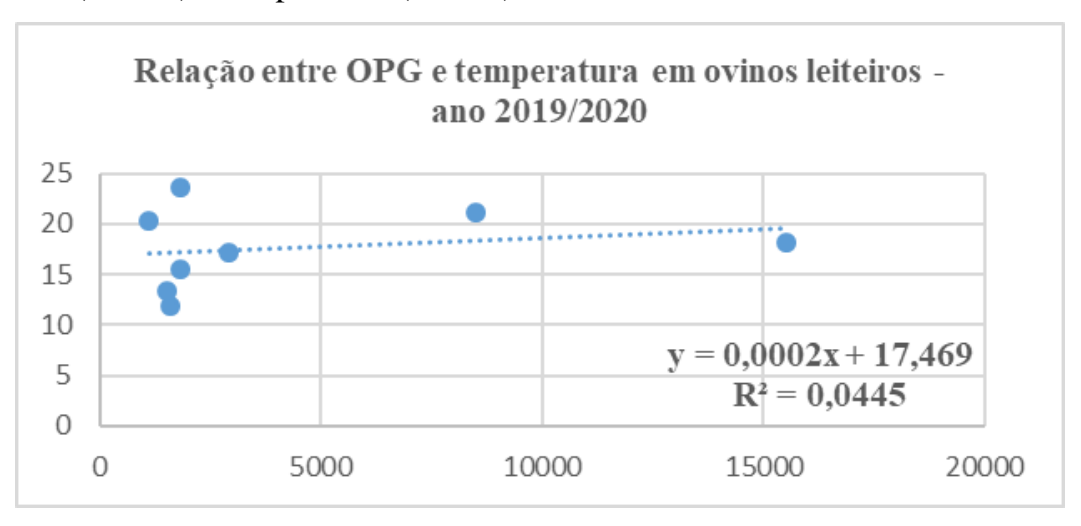

Fonte: Autores.

Como observado no gráfico acima, o índice de correlação entre temperatura média para os 45 dias que antecederam a coleta e o OPG inicial médio foi de 0,0445. Com isso, a temperatura não parece ter influência direta no desenvolvimento destes parasitas, conforme estes resultados. Este fato está em concordância com o relatado por Pimentel (1976) e Heck (2005), em que a temperatura média ideal ao desenvolvimento de larvas infectantes é, em média, $17^{\circ} \mathrm{C}$. Porém, temperaturas oscilantes entre 12 e $37^{\circ} \mathrm{C}$, propiciam condições para sobrevivência de estádios pré-infectantes. Portanto, por ter um limiar amplo, acredita-se que esse fator climático não atua como um interferente direto ao desenvolvimento destes parasitas.

Quanto às análises de coprocultura, encontra-se, na Tabela 2, um resumo dos resultados:

Tabela 2. Resultados percentuais (\%) da prevalência dos gêneros obtidos no exame de coprocultura, de acordo com as estações do ano.

\begin{tabular}{lcccc}
\hline Estações do Ano & Haemonchus contortus & Trichostrongylus spp. & Cooperia spp. & Ostertagia spp. \\
\hline Outono & 51,5 & 43,5 & 2 & 2,5 \\
\hline Inverno & 46,6 & 43,5 & 11,5 & - \\
\hline Primavera & 59,5 & 16 & 24,5 & - \\
\hline Verão & 79 & 12 & 9 & - \\
\hline
\end{tabular}

Fonte: Autores.

Pode-se observar nestes dados, os seguintes nematódeos em cada estação do ano: outono - 51,5\% de Haemonchus contortus; 43,5\% de Trichostrongylus spp.; 2,5\% de Ostertagia spp. e 2,0\% de Cooperia spp.; Inverno: 46\% de Haemonchus contortus; 43,5\% de Trichontrongylus spp; 11,5\% de Cooperia spp; Primavera - 59,5\% de Haemonchus contortus; $24,5 \%$ de Cooperia spp e 16\% de Trichostrongylus spp; Verão - 79\% de Haemonchus contortus; $12 \%$ de Trichostrongylus spp e $9 \%$ de Cooperia spp. Havendo, portanto, conforme esses dados, predominância dos nematódeos gastrointestinais dos gêneros Haemonchus sp. e Trichostrongylus sp. Esses resultados vão de encontro aos obtidos por Lima et al. (2010), no estado de Pernambuco e por Rosalinski-Moraes (2007), no Oeste de Santana Catarina, em que as prevalências das infecções são predominantemente causadas por Haemonchus contortus, seguido por Trichostrongylus. Na Bahia, em estudo realizado por Quadros et al. (2010), o gênero Haemonchus foi o mais prevalente, assim como ocorreu no decorrer de todas amostragens ao longo do ano do presente estudo. De acordo com Amarante et al. (2014), no Rio Grande do Sul, a exemplo dos demais estados brasileiros, H. contortus é a espécie mais predominante e patogênica em ovinos. Embora, este nematódeo gastrointestinal possa ser encontrado o ano todo, sua predominância se faz maior quando a temperatura está acima dos $18^{\circ} \mathrm{C}$ e a umidade elevada 
(80\%), sendo, portanto, fatores cruciais para o desenvolvimento das fases de vida livre (Pegoraro et al., 2008, Andriola et al., 2011). Acredita-se que, por isso, quando as temperaturas ficaram mais baixas na localidade pesquisada, no caso, outono e inverno, o gênero Trichonstrogylus sp., teve maior prevalência, quase em igualdade à espécie $H$. contortus (Pegoraro et al., 2008). Portanto, observou-se, por meio destas análises coprológicas, que nesta propriedade, mesmo nos meses de temperaturas mais baixas, H. contortus sempre foi o mais prevalente, tendo Trichostrongylus spp. e Ostertagia, uma ocorrência mais expressiva quando as temperaturas foram menores. Cabe destacar que o gênero Ostertagia, ocorre apenas no Sul do Brasil, em decorrência, justamente, das temperaturas mais baixas, que favorecem ao seu desenvolvimento, não sendo registrada em ovinos criados em outras regiões do país (Amarante et al, 2014). Isso justifica o achado na presente pesquisa, em que houve registro apenas no outono, quando as temperaturas na localidade se enquadraram ao perfil ambiental característico para ocorrência deste gênero. $\mathrm{O}$ fato de Haemonchus contortus ter sido o mais prevalente, provavelmente, influenciou na resistência a maioria dos anti-helmínticos testados (Melo et al., 2003), conforme pode ser visualizado na tabela 3. Acredita-se que isso tenha ocorrido, uma vez que esse nematódeo desenvolve resistência mais rapidamente devido ao seu alto potencial biótico (Echevarria \& Trindade, 1989), grande variabilidade genética e por albergar o alelo que causa a diminuição da susceptibilidade à uma droga (Blackhall et al., 1998). A fase ambiental inicia-se com a liberação dos ovos nas pastagens, por meio das fezes, e posterior liberação das larvas que se desenvolvem até o estágio infectante (L3), em condições ideais, como temperatura entre 18 a $26^{\circ} \mathrm{C}$ e umidade entre 80 a $100 \%$ (Climeni et al., 2008).

O resultado completo, obtido por meio de testes de Redução na Contagem de Ovos por gramas de fezes (RCOF) para cada fármaco utilizado ao longo deste experimento, podem ser visualizados na tabela abaixo.

Tabela 3. Média do número de ovos por grama de fezes (OPG) de nematódeos gastrintestinais nos dias 0 (pré-tratamento) e dia 7 (pós-tratamento) e percentual médio da eficácia de drogas anti-helmínticas (RCOF) em ovinos da propriedade estudada.

\begin{tabular}{lccc}
\hline \multicolumn{1}{c}{ Princípios ativos } & OPG D0 & OPG D7 & RCOF \\
\hline Nitroxinil + Ivermectina & 1100 & 500 & $54 \% \mathrm{~b}$ \\
\hline Disofenol & 2900 & 1700 & $41 \% \mathrm{c}$ \\
\hline Levamisole & 1800 & 700 & $61 \% \mathrm{c}$ \\
\hline Monepantel & 1600 & 100 & $93 \% \mathrm{a}$ \\
\hline Albendazole & 1500 & 600 & $60 \% \mathrm{c}$ \\
\hline Closantel & 15500 & 2100 & $86 \% \mathrm{~b}$ \\
\hline Rafoxanide & 8500 & 800 & $90 \% \mathrm{a}$ \\
\hline Moxidectina & 1800 & 300 & $84 \% \mathrm{~b}$ \\
\hline
\end{tabular}

Legenda: D0= Dia 0; D7= Dia 7; RCOF: Redução da Contagem de Ovos nas Fezes; Valores seguidos pela mesma letra não diferem estatisticamente entre si $(\mathrm{P} \leq 0,05)$.

Fonte: Autores.

Em relação ao RCOF, observou-se que, a maioria dos fármacos do estudo, apresentou baixa capacidade de redução da infecção parasitária, sendo obtidos os seguintes resultados: Disofenol, Nitroxinil + Ivermectina, Albendazol, Levamisole, Moxidectina e Closantel apresentaram taxas de 41\%, 54\%, 60\%, 61\%, 77\%, 84\% e 86\%, respectivamente. A eficácia abaixo de $90 \%$ é suficiente para pressupor a resistência dos helmintos a essas drogas (Chagas \& Veríssimo, 2008). Portanto, é possível afirmar, a partir destes dados, que há resistência múltipla na propriedade estudada.

Os fármacos Rafoxanide e Monepantel apresentaram capacidade de redução da infecção parasitária em 90\% e 93\%, respectivamente, sendo os únicos a atenderem à taxa de eficácia. Quanto ao Monepantel, acredita-se que isso se deva por 
tratar-se do fármaco mais recentemente introduzido no mercado, tendo sua comercialização liberada a partir de 2012 pela Anvisa, no Brasil. Este caracteriza-se como um composto derivado da aminoacetonitrila (Kaminsky et al., 2008), pertence à uma categoria de fármacos cujo mecanismo de ação é distinto dos demais, o que preconiza sua capacidade de eficácia. Porém, já existem relatos de resistência ao mesmo, quando usado por períodos subsequentes e como único fármaco de controle em uma mesma propriedade (Mederos et al., 2014; Martins, 2016). Quanto a taxa de redução obtida para rafoxanide, pressupõe-se que tenha se dado pelo seu uso durante o verão, estação em que as características climáticas, conforme já mencionado acima, favorecem à prevalência acentuada de $H$. contortus (Amarante et al, 2014). Isso pode ter sido decisivo ao resultado encontrado, pois trata-se de um fármaco com ação mais específica, sendo eficaz, apenas frente ao Haemonchus contortus, além de formas adultas e jovens de Fascíola e larvas de Oestrus ovis.

\section{Considerações Finais}

Os dados de OPG indicaram que o ápice de produção de ovos foi na primavera. Já com os resultados obtidos nas análises coprológicas, constatou-se a prevalência do parasito Haemonchus contortus durante todas as estações do ano em que ocorreu este estudo, com índices de prevalência ainda maiores na primavera.

Além disso, observou-se resistência dos helmintos à grande maioria dos fármacos utilizados para controle das endoparasitoses gastrointestinais nesta propriedade, indicando a necessidade de estudos que viabilizem o controle desta problemática estabelecida na ovinocultura, inclusive, incentivar a busca por novas moléculas ativas frente a estes helmintos.

\section{Agradecimentos}

Ao CNPq, pela bolsa produtividade (312329/2012-9) do orientador da pesquisa, Velci Queiroz de Souza.

À Universidade Estadual do Rio Grande do Sul (UERGS), por permitir a realização deste estudo, especialmente aos membros do Projeto Ovinos da Unidade de Santana do Livramento, pela colaboração no mesmo.

\section{Referências}

Amarante, A. F. T., Bassetto, C. C., Silva, B. F. \& Fernandes, S. (2009). Contaminação da pastagem com larvas infectantes de nematoides gastrintestinais após o pastejo de ovelhas resistentes ou susceptíveis à verminose. Rev. Bras. Parasitol. Vet., Jaboticabal, 18(4), 63-68. 10.4322/rbpv.01804012

Amarante, A. F. T., Ragozo, A. M. A. \& Silva, B. F. (2014). Os parasitos de ovinos. 266p. São Paulo: Editora Unesp Digital. http://editoraunesp.com.br/catalogo/9788568334423,os-parasitas-de-ovinos.

Aquino, R. S., Lemos, C. G., Alencar, C. A., Silva, E. G., Silva, L. R., Gomes, J. A. F. \& Silva, A. F. (2016). A realidade da caprinocultura e ovinocultura no semiárido brasileiro: um retrato do sertão do Araripe, Pernambuco. PUBVET, 10, 271-281. https://.org/10.22256/pubvet.v10n4.271-281

Buriol, G. A., Estefanel, V., Chagas, A. C. \& Kuinchtner, A. (2019). Relação da vegetação natural do Estado do Rio Grande do Sul com as disponibilidades climáticas. Ciênc. $\quad$ Florest., Santa Maria, 29(1), 233-242. http://www.scielo.br/scielo.php?script=sci_arttext\&pid=S1980$50982019000100233 \& \operatorname{lng}=$ en\&nrm=iso

Braga, R. M. (1986). Sobrevivência de larvas infectantes de nematódeos gastrintestinais de bovinos, sob condições naturais. Revista Brasileira de Medicina Veterinária, 8, 186- 188. https://www.scielo.br/scielo.php?script=sci_nlinks\&ref=000048\&pid=S0103-8478200500060003900002\&lng=pt

Cardoso, M. V., Pino, F. A., Federsoni, I. F. P., Filho, A. L. \& Felicio, A. L. (2015). Caracterização da caprinocultura e ovinocultura no estado de São Paulo. Arquivo do Instituto Biológico, São Paulo, 82, 1-15. https://.org/10.1590/1808-1657000592013

Cezar, A. S., Vogel, F. S. F., Sangioni, L. A., Antonello, A. M., Camillo, G., Toscan, G. \& Araujo, L. O. (2010). Ação anti-helmíntica de diferentes formulações de lactonas macrocíclicas em cepas resistentes de nematódeos de bovinos. Pesquisa veterinária brasileira, 30, 523-528. https://www.scielo.br/pdf/pvb/v30n7/a02v30n7.pdf

Costa, P. T., Costa, R. T. \& Vaz, R. Z. (2017). Eficácia anti-helmíntica comparativa do nitroxinil, levamisol, closantel, moxidectina e fenbendazole no controle parasitário em ovinos. Boletim de Indústria Animal, 74(1), 72-78. https://.org/10.17523/bia.v74n1p72.

Domingues, L. F., Giglioti, R., Feitosa, K. A., Fantatto, R. R., Rabelo, M. D., De Sena Oliveira, M. C., Bechara, G. H., De Oliveira, G.P., Barioni Junior, W. \& De Souza Chagas, A. C. (2013). .In vitro and in vivo evaluation of the activity of pineapple (Ananas comosus) on Haemonchus contortus in Santa Inês sheep. Vet Parasitol. 197(1-2):263-70. 10.1016/j.vetpar.2013.04.031. 
Gordon, II. Mc. \& Whitlock, H. V. A new technique for counting nematode eggs in sheep facces. (1939). J. Coune. Indust Res., 12(1):50-2. http://hdl.handle.net/102.100.100/339340?index=1

Heck, I., Leandro, A. S., Leite, C. T., Gindri, J. K., Souza, M. B. M., Depner, R. \& Molento, M. B. (2005). Efeito do clima sobre a infecção parasitária em bezerros e presença de larvas em manejo rotativo de pasto em Santa Maria, RS, Brasil. Ciência Rural, Santa Maria, 35(6), 1461-1464. https://.org/10.1590/S0103-84782005000600039

IBGE. (2018). Censo Agropecuário 2017. Instituto Brasileiro de Geografia e Estatística, https://biblioteca.ibge.gov.br/visualizacao/periodi cos/3093/agro_2017_resultados_preliminares.pdf.

Kate, R.C. (1965). Ecological aspects of helminth transmission in domesticated animals. Am. Zoologist 5:95-130. In: Fonseca, H. A. \& Neto, M. P. Epidemiologia das helmintoses pulmonares e gastrintestinais Epidemiologia das helmintoses pulmonares e gastrintestinais de bezerros em região de baixada do Estado do Rio de Janeiro. Pesq. Vet. Bras. 22(4):148-152, 2002. https://www.scielo.br/pdf/pvb/v22n4/14288.pdf

Keith, R. K. (1953). The diferentiation of the infective larvae of some commom nematode. Australian Journal of Zoology, Victoria, 1(2), 223-235.

Lacerda, M. J. R., Rocco, V. V. B., Guimarães, K. C., Souza, P. P. S. \& Fernandes, L. H. (2010). Routes of applying Closantel as a gastrointestinal anthelmintic in sheep. Archivos Latinoamericanos de Producción Animal, 17, 55-59, In: Silva, D. G., Pilatti, J. A., Menezes, B. M., Brum, L. P., Netto, C. G. \& Martins, A. A. (2017). Eficácia anti-helmíntica comparativa entre diferentes princípios ativos em ovinos jovens. PUBVET, 11(4), 356-362. http://www.pubvet.com.br/artigo/3748/eficaacutecia-anti-helmiacutentica-comparativa-entre-diferentes-princiacutepios-ativos-em-ovinos-jovens

Leme, M. E., Peixoto, E. C. T. M., Yoshihara, E., Kakimori, M. T. A., Portes, E. A., Paiva, M. M. \& Silva, M. A. A. (2020). Ação in vitro do extrato do botão floral da bananeira (Musa spp.) sobre nematódeos gastrintestinais de ovinos. Research, Society and Development, 9(8), e965986594. https://.org/10.33448/rsdv9i8.6594

Lopes, B., Ribeiro, A., Correia, L., Camilo, D. J. \& Cesco, G. (2016). Levantamento de parasitas gastrointestinais em ovinos sob lotação continua. Synergismus Scyentifica UTFPR, 11, 43-46. http://revistas.utfpr.edu.br/pb/index.php/SysScy/article/view/2093/1502

Martins, A. C. (2016). Estudo de resistência anti-helmíntica ao monepantel em propriedades de ovinos de uma microrregião em torno de Jaboticabal-SP. Tese (Doutorado - Área de Concentração em Medicina Veterinária) - Faculdade de Ciências Agrárias e Veterinárias, Universidade Estadual Paulista (UNESP), Campus de Jaboticabal. https://repositorio.unesp.br/bitstream/handle/11449/134368/martins_ac_dr_jabo.pdf?sequence=3

Mederos, A. E., Ramos, Z. \& Banchero, G. E. (2014). First report of monepantel Haemonchus contortus resistance on sheep farms in Uruguay. Parasites \& Vectors, 7, 598. https://parasitesandvectors.biomedcentral.com/articles/10.1186/s13071-014-0598-Z

Mena, E. O. G. (2017). A silvicultura uruguaia e o índice pluviométrico do município de Dom Pedrito. Trabalho de conclusão de curso (Licenciatura em Ciências da Natureza) - Universidade Federal do Pampa (UNIPAMPA), Campus Dom Pedrito. http://cursos.unipampa.edu.br/cursos/cienciasdanaturezadp/files/2018/03/a-silvicultura-uruguaia-e-o-indice-pluviometrico-do-municipio-de.pdf

Menezes, L. M., Amaral, F. P., Zardin, M., Farias, G.D., Farias, L. B., Araújo, F. B., Barbosa silveira, I.D. \&Silva, S. S. (2011). Caracterização de resistência anti-helmíntica a moxidectina a $1 \%$ em ovinos criados no Sul do Brasil. Congresso Brasileiro de Medicina Veterinária. Anais..In: CD-Room..Florianópolis. http://www.sovergs.com.br/BKP/site_/38conbravet/resumos/980.pdf

Mottin, V. D, Cruz, J. F., Teixeira Neto, M. R., Marisco, G., Figueredo, J. S. \& Sousa, L. S. (2019). Efficacy, toxicity, and lethality of plants with potential anthelmintic activity in small ruminants in Brazil. Rev. bras. saúde prod. anim., 20, e0232019. http://www.scielo.br/scielo .php?script=sci_arttext\&pid=S1519-99402019000100806\&lng=en\&nrm=iso

Nova, L. E. V., Costa, M. E., Melo, P. G. C. F., Cunha Filho, L. F. C., Junior, F. A. B., Silva, L. C., Okano, W. \& Bogado, A. L. G. (2014). Resistência de nematoides aos anti-helmínticos nitroxinil 34\% e ivermectina 1\% em rebanho ovino no município de São João do Ivaí, Paraná. Revista Brasileira de Higiene e Sanidade Animal, 8, 159-171. http://.org/10.5935/1981-2965.20140011

Osório, T. M., Menezes, L. M., Rosa, K. B., Escobar, R. F., Santos, R. M. L., Maydana, G. M. \& Souza, V. Q. (2020). Resistência anti-helmíntica em nematódeos gastrointestinais na ovinocultura: uma revisão. Braz. J. of Develop, Curitiba, 6(11), 89194-89205. 10.34117/bjdv6n11-368

Pimentel Neto, M. (1976). Epizootiologia da haemoncose em bezerros de gado de leite no estado do Rio de Janeiro. Pesquisa Agropecuária Brasileira, 11, $101-114$.

Pereira A. S., Shitsuka, D. M., Pereira, F. J. \& Shitsuka, R. (2018). Metodologia da pesquisa científica. UFSM. https://repositorio.ufsm.br/bitstream/hand le/1/15824/Lic_Computacao_Metodologia-Pesquisa-Cientifica.pdf?sequence=1

Ramos, C. I., Bellato, V., Souza, A. P., Avila, V. S., Coutinho, G. C. \& Dalagnoll, C. A. (2004). Epidemiologia das helmintoses gastrintestinais de ovinos no Planalto Catarinense. Ciência Rural, 34(6), 1889- 1895. https://www.scielo.br/pdf/cr/v34n6/a34v34n6.pdf

Roberto, F. F. S., Difante, G. S., Zaros, L. G. \& Gurgel, A. L. C. (2018). Nematoides gastrintestinais na ovinocultura de corte sob regime de pastejo. PUBVET. 12(4), a65, pa1-12. file:///C:/Users/Cliente/Downloads/nematoides-gastrintestinais-na-ovinocult\%20(1).pdf

Rocco, V. V. B., Lacerda, M. J. R., Fernandes, L. H., Souza, P. P. S. \& Guimarães, K. C. (2012). Diferentes princípios ativos no controle de helmintos gastrintestinais em ovinos. Global Science and Technology, 5, 194-200.

Salgado, J. A. \& Santos, C. P. (2016). Overview of anthelmintic resistance of gastrointestinal nematodes of small ruminants in Brazil. Rev. Bras. Parasitol. Vet., Jaboticabal, v. 25, n. 1, p. 3-17. http://www.scielo.br/scielo.php?script=sci_arttext\&pid=S1984-29612016000100003\&lng=en\&nrm=iso

Silva, D. G., Menezes, B. M., Bettencourt, A. F., Frantz, A. C., Corrêa, M. R., Ruszkowski, G., Martins, A. A., Brum, L. P. \& Hirschmann, L. C. (2017). Método FAMACHA® como ferramenta para verificar a infestação parasitária ocasionada por Haemonchus spp. em ovinos. PUBVET, 11, 1015-1021 http://www.pubvet.com.br/artigo/3748/eficaacutecia-anti-helmiacutentica-comparativa-entre-diferentes-princiacutepios-ativos-em-ovinos-jovens 
Research, Society and Development, v. 10, n. 3, e34410313315, 2021

(CC BY 4.0) | ISSN 2525-3409 | DOI: http://dx.doi.org/10.33448/rsd-v10i3.13315

Souza, M. F. (2013). Recuperação de larvas infectantes, carga parasitária e desempenho de cordeiros terminados em pastagens com distintos hábitos de crescimento. 107f. Dissertação (Mestrado) - Curso de Pós-graduação em Zootecnia, Universidade Federal do Rio Grande do Sul. 107 f. https://www.lume.ufrgs.br/bitstream/handle/10183/72786/000886123.pdf?sequence=1

Tariq, K. A., Chisthi, M. Z., Ahmad, F. \& Shawl, A. S. (2008). Evaluation of anthelmintic activity of Iris hookeriana against gastrointestinal nematodes of sheep. Journal of Helminthology, 82, 135-141. https://.org/10.1017/S0022149X08912360

Vieira, L.S. (2008). Métodos alternativos de controle de nematoides gastrintestinais em caprinos e ovinos. Revista Tecnologia \& Ciência Agropecuária. 2, 2831. https://ainfo.cnptia.embrapa.br/digital/bitstream/item/42340/1/AAC-Metodos-alternativos.pdf

Vieira, L. S., Cavalvante, A. C. R. \& Zaros, L. G. (2011). Manejo sanitário de doenças parasitárias. Embrapa Caprinos e Ovinos, 15, 355-383. https://www.alice.cnptia.embrapa.br/alice/bitstream/doc/924341/1/PLManejosanitario.pdf 